Case Report

\title{
Concomitant Alpha- and Gamma-Sarcoglycan Deficiencies in a Turkish Boy with a Novel Deletion in the Alpha-Sarcoglycan Gene
}

\author{
Gulden Diniz, ${ }^{1}$ Hulya Tosun Yildirim, ${ }^{2}$ Sarenur Gokben, ${ }^{3}$ Gul Serdaroglu, ${ }^{3}$ \\ Filiz Hazan, ${ }^{4}$ Kanay Yararbas, ${ }^{5,6}$ and Ajlan Tukun ${ }^{5,6,7}$ \\ ${ }^{1}$ Neuromuscular Diseases Centre, Tepecik Research Hospital, Kibris Sehitleri Caddesi 51/11, Alsancak, 35220 Izmir, Turkey \\ ${ }^{2}$ Pathology Department, Dr. Behcet Uz Children's Research Hospital, 35210 Izmir, Turkey \\ ${ }^{3}$ Pediatric Neurology Department, Faculty of Medicine, Ege University, 35100 Izmir, Turkey \\ ${ }^{4}$ Medical Genetics Department, Dr. Behcet Uz Children's Research Hospital, 35210 Izmir, Turkey \\ ${ }^{5}$ Medical Genetics Department, Duzen Laboratories, Istanbul, Turkey \\ ${ }^{6}$ Medical Genetics Department, Duzen Laboratories, Ankara, Turkey \\ ${ }^{7}$ Medical Genetics Department, Faculty of Medicine, Ankara University, 06100 Ankara, Turkey
}

Correspondence should be addressed to Gulden Diniz; agdiniz@gmail.com

Received 21 April 2014; Accepted 2 June 2014; Published 22 June 2014

Academic Editor: Patrick Morrison

Copyright ( $\odot 2014$ Gulden Diniz et al. This is an open access article distributed under the Creative Commons Attribution License, which permits unrestricted use, distribution, and reproduction in any medium, provided the original work is properly cited.

Limb-girdle muscular dystrophy type 2D (LGMD-2D) is caused by autosomal recessive defects in the alpha-sarcoglycan gene located on chromosome 17q21. In this study, we present a child with alpha-sarcoglycanopathy and describe a novel deletion in the alpha-sarcoglycan gene. A 5-year-old boy had a very high serum creatinine phosphokinase level, which was determined incidentally, and a negative molecular test for the dystrophin gene. Muscle biopsy showed dystrophic features. Immunohistochemistry showed that there was diminished expression of alpha- and gamma-sarcoglycans. DNA analysis revealed a novel 7 bp homozygous deletion in exon 3 of the alpha-sarcoglycan gene. His parents were consanguineous heterozygous carriers of the same deletion. We believe this is the first confirmed case of primary alpha-sarcoglycanopathy with a novel deletion in Turkey. In addition, this study demonstrated that both muscle biopsy and DNA analysis remain important methods for the differential diagnosis of muscular dystrophies because dystrophinopathies and sarcoglycanopathies are so similar.

\section{Introduction}

Limb girdle muscular dystrophy type $2 \mathrm{D}$ (LGMD-2D) is an autosomal recessive muscular disease caused by genetic defects in sarcolemmal alpha sarcoglycan ( $\alpha$-SGC) glycoprotein. Alpha-SGC or adhalin, one of the four sarcoglycans (SGCs), is essential for membrane integrity during muscle contraction and provides a scaffold for important signaling molecules [1-3]. Alpha-SGC is encoded by the sarcoglycan alpha gene (SGCA) located on chromosome 17q21 [1, 4]. LGMD-2D predominantly affects proximal muscles around the scapular and the pelvic girdles. LGMD-2D has a very heterogeneous phenotype. The age of onset, rate of progression, and the severity of disease can vary between and also within affected families. The most clinically severe course is generally observed when the sarcolemmal $\alpha$-SGC is totally absent whereas milder phenotypes are observed when residual proteins are present [1-4]. Interestingly, a mutation in any SGC gene can lead to a reduction or absence of the other SGCs [4-7]. It was previously reported that the SGCA gene must be evaluated first if there is a concomitant absence of $\alpha$-SGC and gamma- $(\gamma$-) SGC [4].

The differential diagnosis for LGMD-2D includes Duchenne and Becker muscular dystrophies (DMD/BMD) and it is impossible to reach a diagnosis on clinical grounds alone. Therefore, immunohistochemical staining of a muscle biopsy and molecular genetic analysis are mandatory for the correct diagnosis $[3,5,8,9]$. In this report, the patient's 


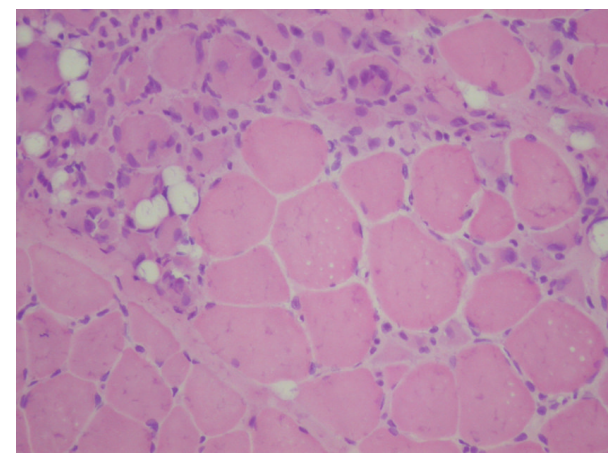

FIGURE 1: Differences in the size and shape of myofibers as well as regeneration (HEx 200).

genotype was a previously unknown $7 \mathrm{bp}$ deletion in exon 3. This finding adds to the growing spectrum of mutations in the alpha-sarcoglycan gene. Finally, we also discuss important considerations in the differential diagnosis of the muscular dystrophies.

\section{Case Report}

A 5-year-old boy had second degree consanguineous parents from Turkey without an ancestral history of neuromuscular disorders. There were no complications during pregnancy, and antenatal signs of muscular disorders such as polyhydramnios and reduced fetal movements were not noted. Cognitive and motor development was normal. At the time of presentation, his previously undetected mild muscle weakness was predominantly proximal. Deep tendon reflexes were present and he had no contractures. He was walking normally but he had mild difficulty when climbing stairs and running. Pulmonary function tests were normal. His creatinine phosphokinase (CPK) levels were between 9000 and 15000 units per liter (normal < $250 \mathrm{U} / \mathrm{L}$ ), and there were myopathic changes on electromyography. Because of the very high CPK level, muscular dystrophy was suspected and, after informed consent, samples were obtained for histopathology, immunohistochemistry, and molecular genetics testing.

A muscle biopsy specimen from the left gastrocnemius muscle of the patient was frozen in isopentane that was precooled to $-160^{\circ} \mathrm{C}$ in liquid nitrogen. Cryosections were immunostained for dystrophin using a polyclonal antibody (Neomarkers), with a monoclonal spectrin antibody (Novocastra) as a control. A neonatal myosin heavy chain (Neonatal myosin, Novocastra) antibody was used for the identification of pathological immature myofibers. SGCs were detected with anti- $\alpha-,-\beta-,-\delta-$, and $-\gamma$-SGC antibodies (Novocastra).

Peripheral blood specimens were collected from the proband and parents. Genomic DNA was extracted from whole blood using a commercial DNA extraction kit (QiaGen, USA) following the standard manufacturer's protocol. The concentration of sample DNA was determined by a NanoDrop spectrophotometer (NanoDrop Technologies, Wilmington, DE). The exon regions and flanking short intronic sequences of the SGCA gene were amplified using polymerase

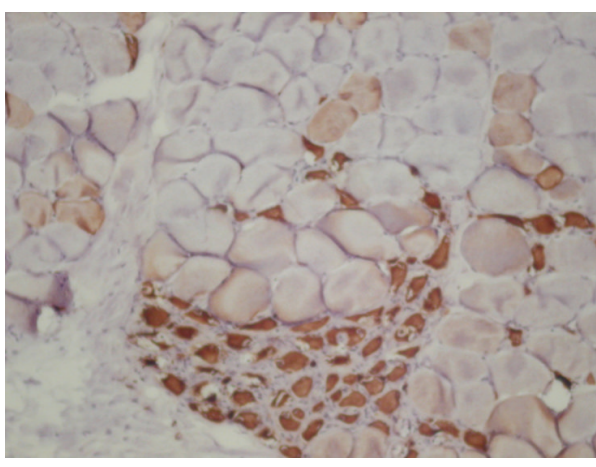

FIGURE 2: Immature pathological fibers visualized with antineonatal myosin antibody staining (DABx 100).

chain reaction (PCR), followed by direct sequencing of the PCR products (ABI, US) (NCBI Reference Sequence: NG_008889.1). Hitherto reported genetic abnormalities in LGMD-2D are listed in Table 1.

\section{Results}

The muscle biopsy showed dystrophic changes like contraction, regeneration (Figure 1), degeneration, necrosis, nuclear internalization, and fibrosis. In addition, many pathological immature myofibers were visualized using the neonatal myosin staining (Figure 2). Based on immunostaining, dystrophin and spectrin expressions were normal. Except for isolated deficient fibers, beta $(\beta)$ sarcoglycan and delta $(\delta)$ sarcoglycan were present at normal levels, whereas $\alpha$-SGC and $\gamma$-SGC were diffusely absent (Figure 3 ).

Based on analysis of the proband, we have identified a previously undetermined homozygous $7 \mathrm{bp}$ deletion in exon 3 (Figure 4). A similar heterozygous deletion was found in both parents (Figures 5 and 6). Location of this deletion was also indicated in Table 1 . In addition, there were no abnormalities in the dystrophin gene and the other sarcoglycan genes (SGCB, SGCD, and SGCG) in the patient and his parents.

\section{Discussion}

Human SGCA cDNA from a human skeletal muscle library was isolated and sequenced in 1993. This gene consisted of 10 exons. The protein product of SGCA gene consisted of 387 amino acids with an extracellular N-terminus, a transmembrane domain, and an intracellular C-terminus. Northern blot analysis showed that human adhalin mRNA was expressed at the highest levels in skeletal muscle. It was also expressed in cardiac muscle and in the lung, but at much lower levels. On the contrary, adhalin mRNA was not detected in the brain. It was also reported that the adhalin mRNA from cardiac muscle was shorter relative to skeletal muscle and that the base sequence encoding the transmembrane domain was absent. It is known that LGMD-2D primarily affects skeletal muscles while brain and peripheral nerve functions are largely preserved. Briefly, the less severe cardiac dysfunction and lack of mental retardation 


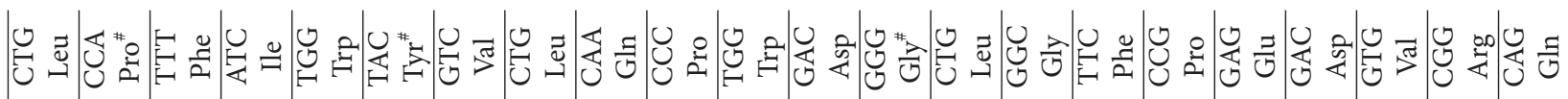

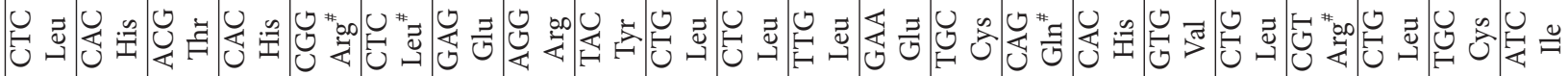

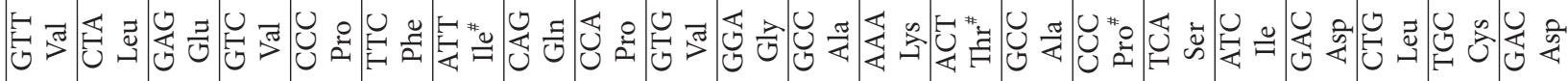

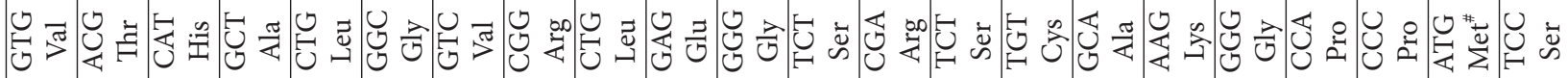

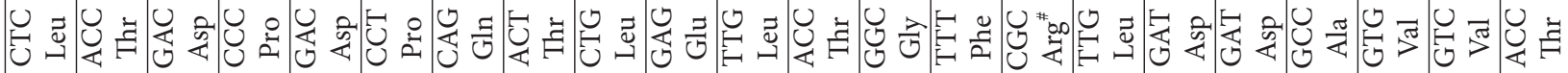

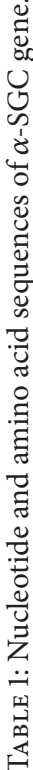

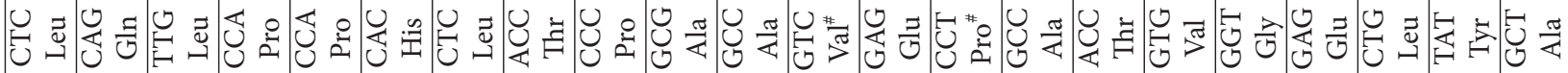

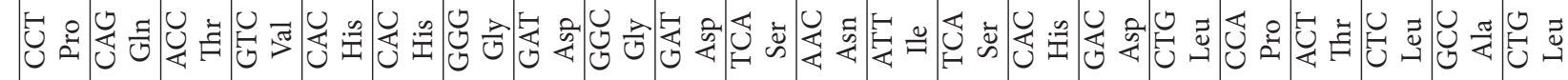

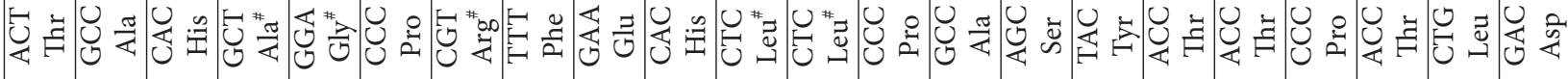

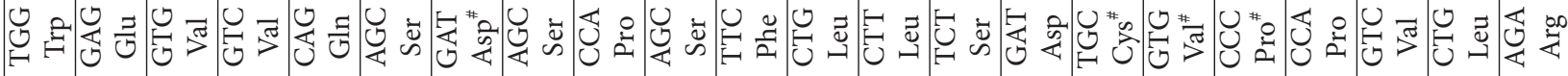

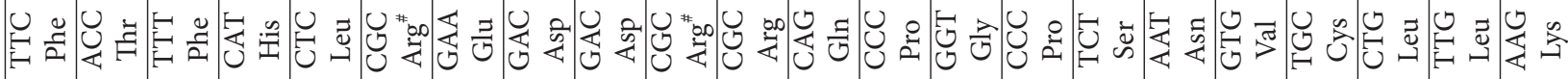

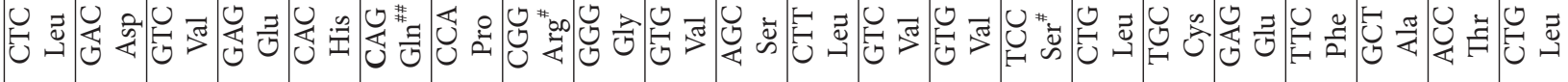

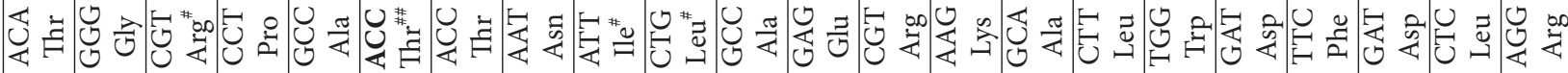

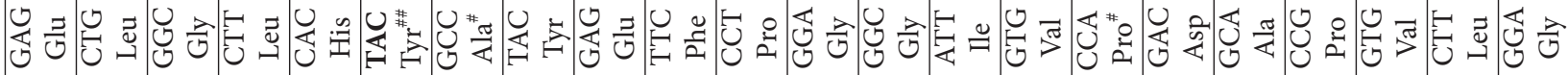

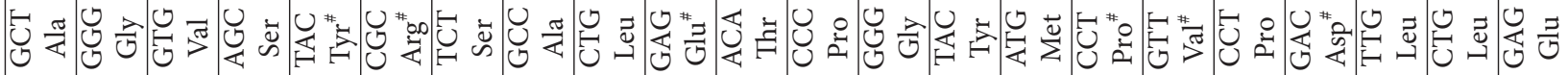

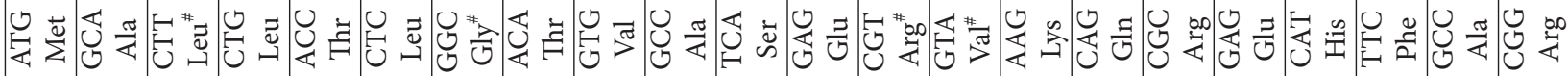

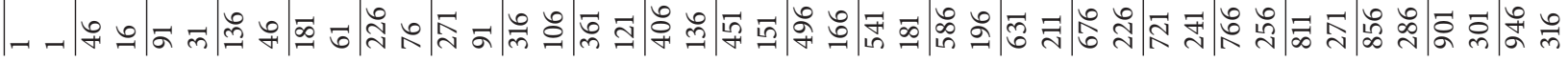




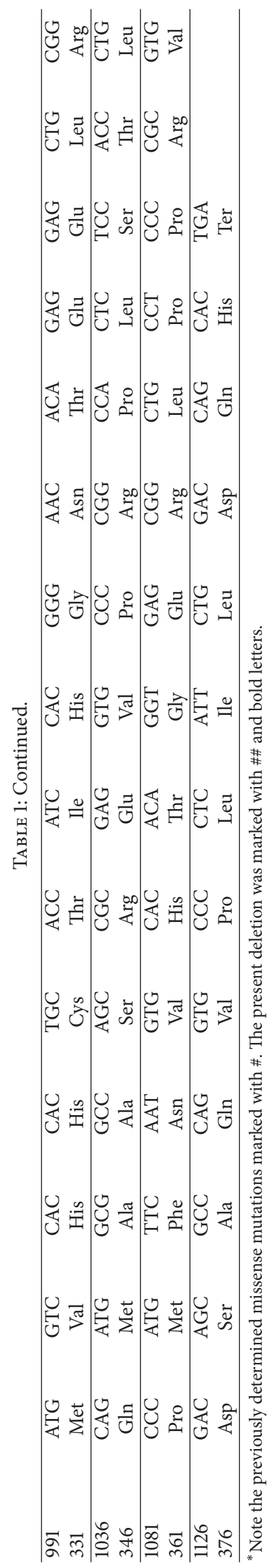




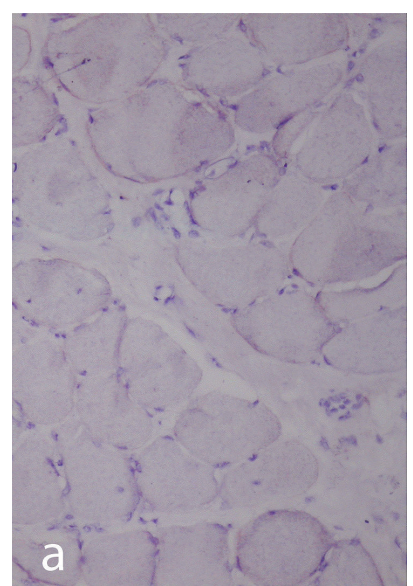

(a)

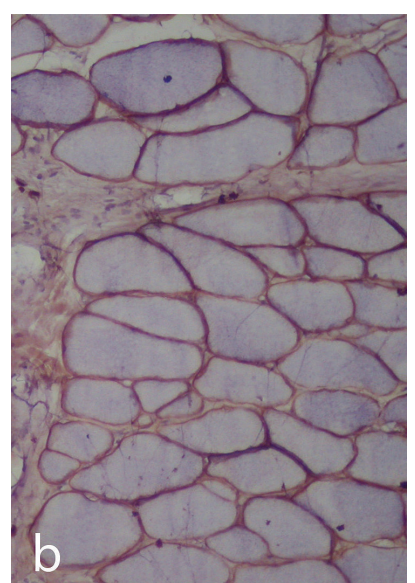

(b)

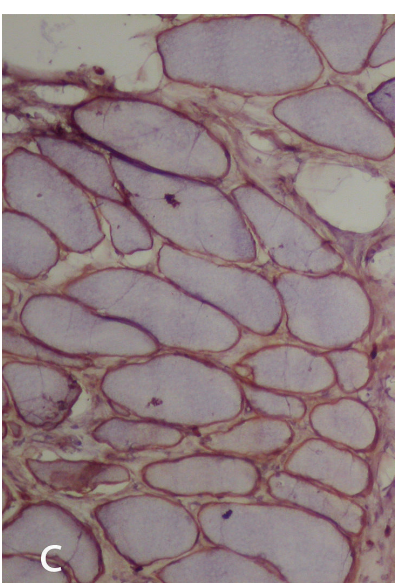

(c)

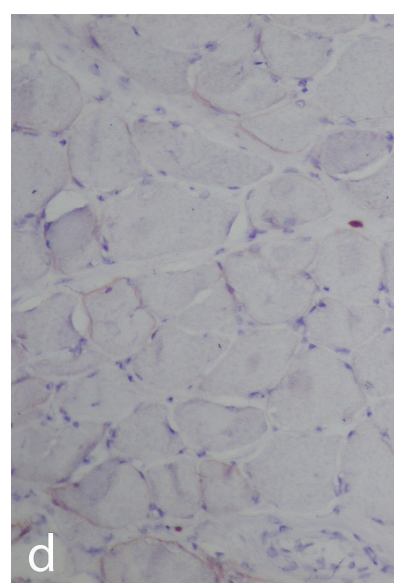

(d)

FIGURE 3: Diffuse absence of sarcolemmal $\alpha$-SGC (a) and $\gamma$-SGC (d) expression and normal $\beta$-SGC (b) and $\delta$-SGC (c) expression (DABx 200).

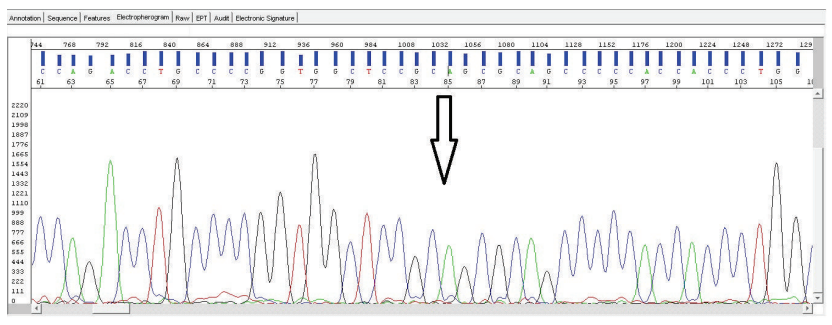

FIgure 4: Proband exon 3 homozygous del TACACCC site.

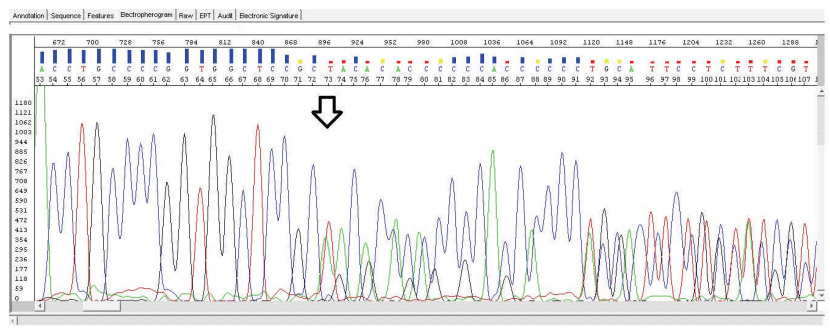

Figure 5: Maternal heterozygous del TACACCC site.

in patients with LGMD-2D may be explained by the lower expression of $\alpha$-SGC in cardiac muscle and the absence of adhalin expression in the brain $[1,3,10]$. In the patient described in this report, we did not find clinical evidence of cardiac involvement, decreased intellectual capacity, or denervation (as demonstrated by electromyography). The course of the disease in this case suggests that this novel deletion may cause a milder phenotype of LGMD-2D despite the diffuse absence of $\alpha$-SGC and $\gamma$-SGC.

Immunohistochemical analysis of sarcolemmal proteins in muscle biopsies like dystrophin, SGCs, merosin, and dysferlin is an important part of the diagnostic evaluation of patients with muscular dystrophy. Reduced or absent sarcolemmal expression of one of the 4 SGCs can be found in patients with any type of LGMDs and also in patients

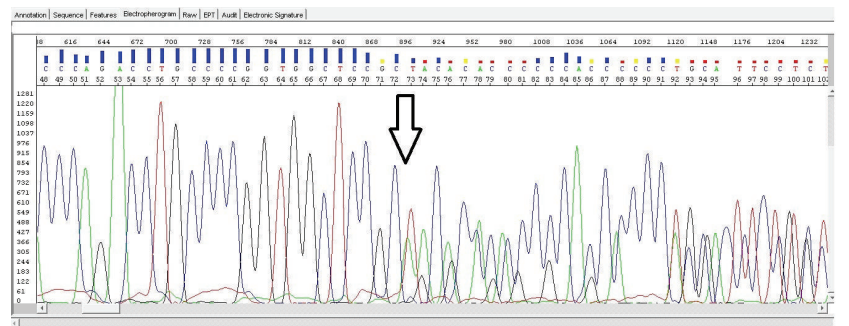

Figure 6: Paternal heterozygous del TACACCC site.

with dystrophinopathies. It has previously been suggested that different patterns of SGC expression could predict the primary genetic defect and that genetic analysis could be directed by these patterns [5-8]. However, Klinge et al. [9] reported that residual SGC expression could be highly variable and an accurate prediction of the genotype could not be achieved. Babameto-Laku et al. [4] also determined that the concomitant absence of $\alpha$-SGC and $\gamma$-SGC expression was caused by defects in the SGCA gene. Therefore, they recommended using antibodies against all four SGCs for immunoanalysis of skeletal muscle sections. Similarly, a concomitant reduction in dystrophin and any of the SGCs may illustrate the importance of considering coexisting dystrophinopathies in patients with sarcoglycan-deficient LGMD [9-13]. For this reason, it is not easy to decide whether the disease is a dystrophinopathy with defective expressions of SGCs or a LGMD with defective expression of dystrophin. However, in the patient described in this report, dystrophinopathies, such as DMD and BMD, were ruled out because the expression of sarcolemmal dystrophin was diffusely present and molecular tests for dystrophin gene were normal.

At present, more than 70 mutations have been reported in the SGCA gene that cause changes in the $\alpha$-SGC glycoprotein. Approximately a two-thirds of mutations are missense mutations that generate a complete protein with a single 
residue substitution, whereas other mutations like nucleotide replacements, duplications, deletions, or insertions produce truncated, incomplete, or anomalous proteins. Almost all missense mutations map to the extracellular domain which is a critical region for the organization of SGCs and their association with dystroglycan. Only a single missense mutation maps to the intracellular domain and causes LGMD2D in homozygous cases. Similarly, two mutations caused by deletions generate a normal extracellular portion of $\alpha$ SGC and truncated intracellular tails. At present, there is no data about the intracellular tail of the $\alpha$-SGC protein and its function $[1,10-14]$. In the family described in this report, we discovered a novel deletion in the TACACCC site of exon 3 that would cause a frame-shift mutation. The past literature highlights that the prediction of pathological consequences associated with different mutations of SGCA gene is very complex. It is not clear whether this novel deletion generates a severe disease phenotype or whether it also has additional, undetermined consequences.

Patients with any of the LGMDs may be clinically indistinguishable from those with the primary dystrophinopathies. It is likely that the prevalence of LGMD is underestimated and a number of male patients are incorrectly diagnosed with DMD or BMD [13]. A definitive diagnosis rests on performing the appropriate immunohistochemical examination as well as doing a molecular analysis. A normal dystrophin staining pattern should be seen as well as an autosomal recessive mode of inheritance. In contrast, the patients with dystrophinopathies may show variable findings from a normal to a regional absence or a mosaic pattern of sarcolemmal staining with anti-SGCs antibodies which correspond to an abnormal organization of the cell-membrane-associated dystrophin glycoprotein complex. Therefore, it is necessary to perform a careful examination of the immunohistochemical staining as well as a genetic study in order to make the correct diagnosis.

In summary, this report describes a novel deletion that adds to the growing list of defects associated with LGMD-2D and further emphasizes the importance of systematic analysis of all related genes, instead of limiting the analysis to the one SGC gene that is hypothesized to be the cause of the abnormalities. In this study, we also highlight the complexity of staining patterns associated with sarcolemmal proteins and the importance of careful analysis of this staining pattern in order to narrow the differential diagnosis of muscular dystrophies.

\section{Conflict of Interests}

The authors declare that there is no conflict of interests.

\section{References}

[1] D. Sandonà and R. Betto, "Sarcoglycanopathies: molecular pathogenesis and therapeutic prospects," Expert Reviews in Molecular Medicine, vol. 11, p. e28, 2009.

[2] A. Carrié, F. Piccolo, F. Leturcq et al., "Mutational diversity and hot spots in the $\alpha$-sarcoglycan gene in autosomal recessive muscular dystrophy (LGMD2D)," Journal of Medical Genetics, vol. 34, no. 6, pp. 470-475, 1997.

[3] B. Eymard, N. B. Romero, F. Leturcq et al., "Primary adhalinopathy ( $\alpha$-sarcoglycanopathy): clinical, pathologic, and genetic correlation in 20 patients with autosomal recessive muscular dystrophy," Neurology, vol. 48, no. 5, pp. 1227-1234, 1997.

[4] A. Babameto-Laku, M. Tabaku, V. Tashko, M. Cikuli, and V. Mokini, "The first case of primary alpha-sarcoglycanopathy identified in Albania, in two siblings with homozygous alphasarcoglycan mutation," Genetic Counseling, vol. 22, no. 4, pp. 377-383, 2011.

[5] V. Dubowitz, C. A. Sewry, and A. Oldfors, Muscle Biopsy: A Practical Approach, Saunders, Philadelphia, Pa, USA, 2013.

[6] I. Higuchi, H. Kawai, Y. Umaki et al., "Different manners of sarcoglycan expression in genetically proven $\alpha$-sarcoglycan deficiency and $\gamma$-sarcoglycan deficiency," Acta Neuropathologica, vol. 96, no. 2, pp. 202-206, 1998.

[7] E. S. Moreira, M. Vainzof, O. T. Suzuki, R. C. Pavanello, M. Zatz, and M. R. Passos-Bueno, "Genotype-phenotype correlations in 35 Brazilian families with sarcoglycanopathies including the description of three novel mutations," Journal of Medical Genetics, vol. 40, no. 2, p. E12, 2003.

[8] R. Pogue, L. V. B. Anderson, A. Pyle et al., "Strategy for mutation analysis in the autosomal recessive limb-girdle muscular dystrophies," Neuromuscular Disorders, vol. 11, no. 1, pp. 80-87, 2001.

[9] L. Klinge, G. Dekomien, A. Aboumousa et al., "Sarcoglycanopathies: can muscle immunoanalysis predict the genotype?" Neuromuscular Disorders, vol. 18, no. 12, pp. 934-941, 2008.

[10] M. Trabelsi, N. Kavian, F. Daoud et al., "Revised spectrum of mutations in sarcoglycanopathies," European Journal of Human Genetics, vol. 16, no. 7, pp. 793-803, 2008.

[11] R. M. Quinlivan, S. A. Robb, C. Sewry, V. Dubowitz, F. Piccolo, and J. C. Kaplan, "Absence of alpha-sarcoglycan and novel missense mutations in the alpha-sarcoglycan gene in a young British girl with muscular dystrophy," Developmental Medicine and Child Neurology, vol. 39, no. 11, pp. 770-774, 1997.

[12] S. Ávila De Salman, A. L. Taratuto, G. Dekomien, and R. Carrero-Valenzuela, "Alpha vs. gamma sarcoglycanopathy: DNA tests solve a case from Argentina," Acta Myologica, vol. 26, no. 2, pp. 115-118, 2007.

[13] U. Schara, M. Gencik, J. Mortier et al., "Alpha-sarcoglycanopathy previously misdiagnosed as Duchenne muscular dystrophy: implications for current diagnostics and patient care," European Journal of Pediatrics, vol. 160, no. 7, pp. 452-453, 2001.

[14] G. Diniz, H. Tosun Yildirim, G. Akinci et al., "Sarcolemmal alpha and gamma sarcoglycan protein deficiencies in Turkish siblings with a novel missense mutation in the alpha sarcoglycangene," Pediatric Neurology, vol. 50, no. 6, pp. 640-647, 2014. 


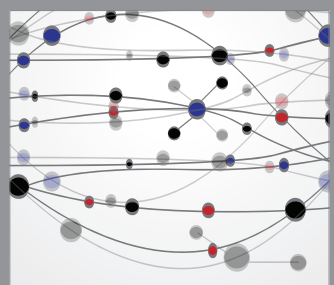

The Scientific World Journal
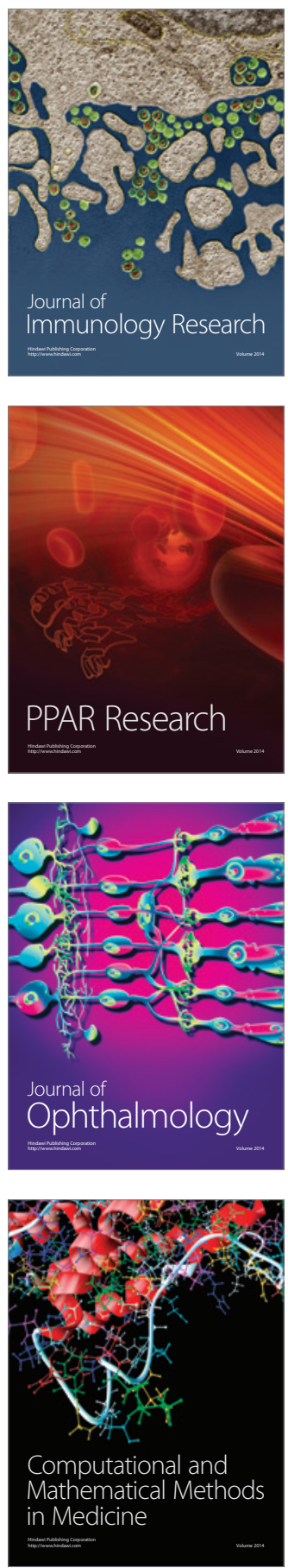

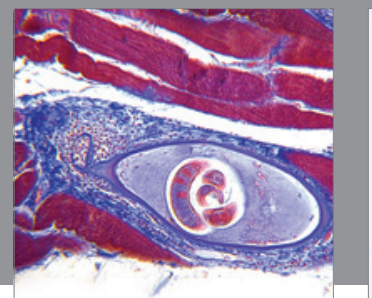

Gastroenterology

Research and Practice
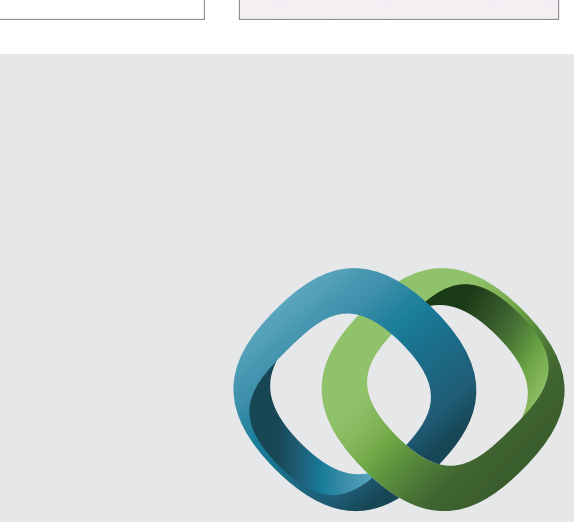

\section{Hindawi}

Submit your manuscripts at

http://www.hindawi.com
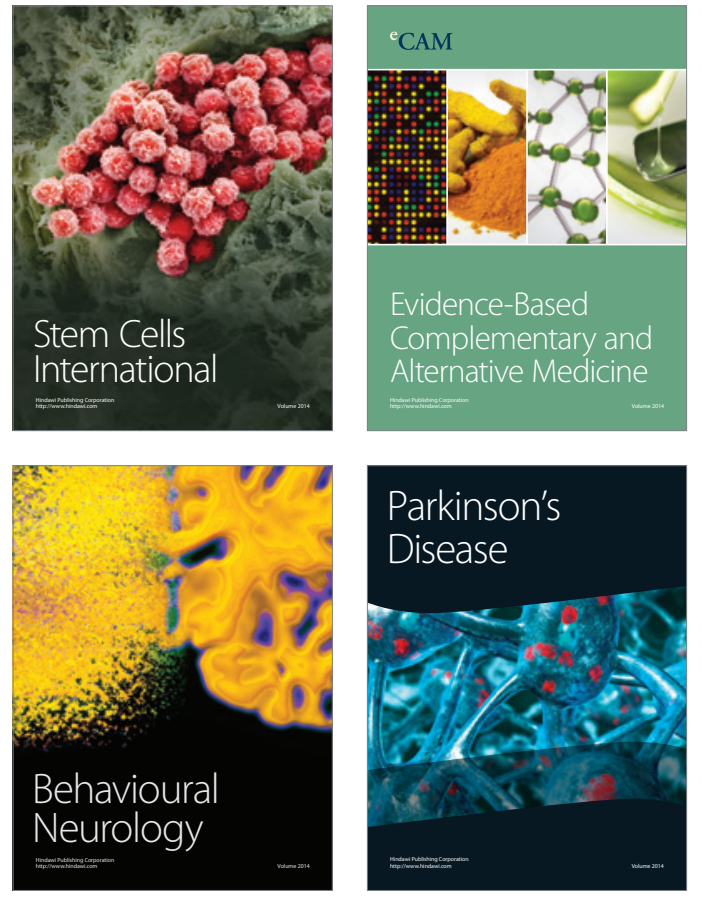
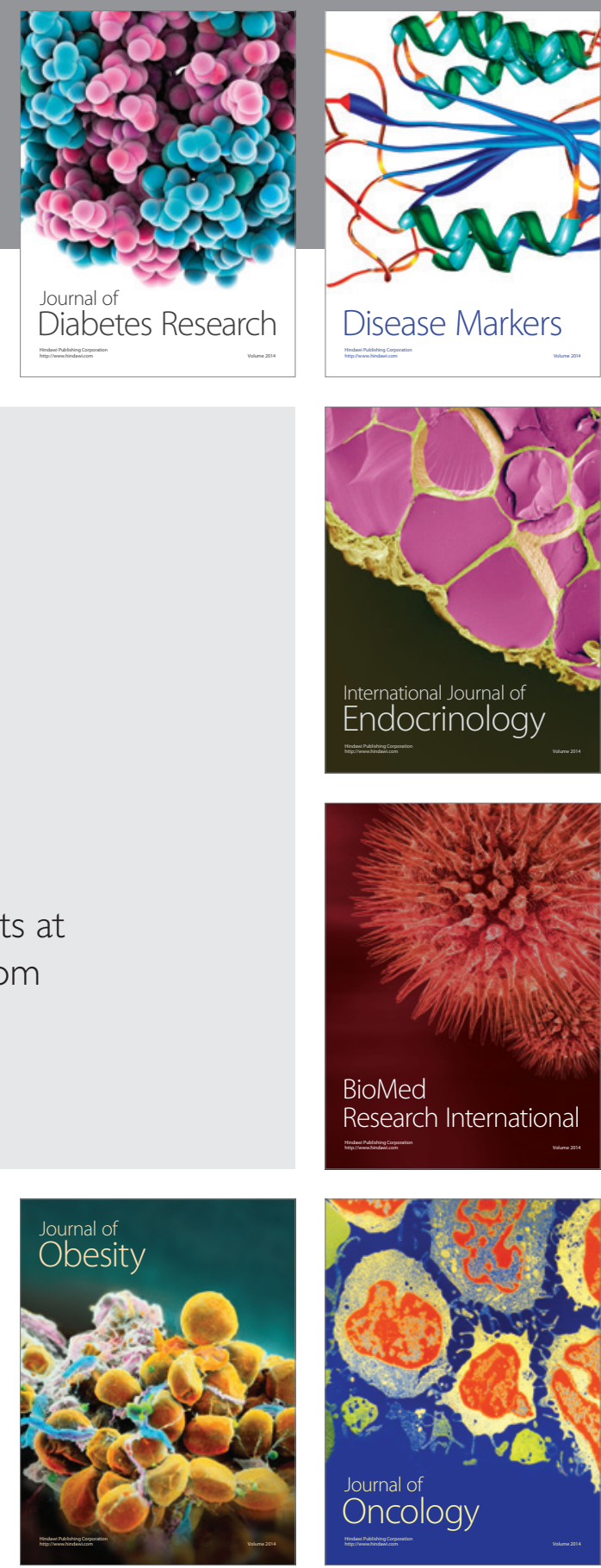

Disease Markers
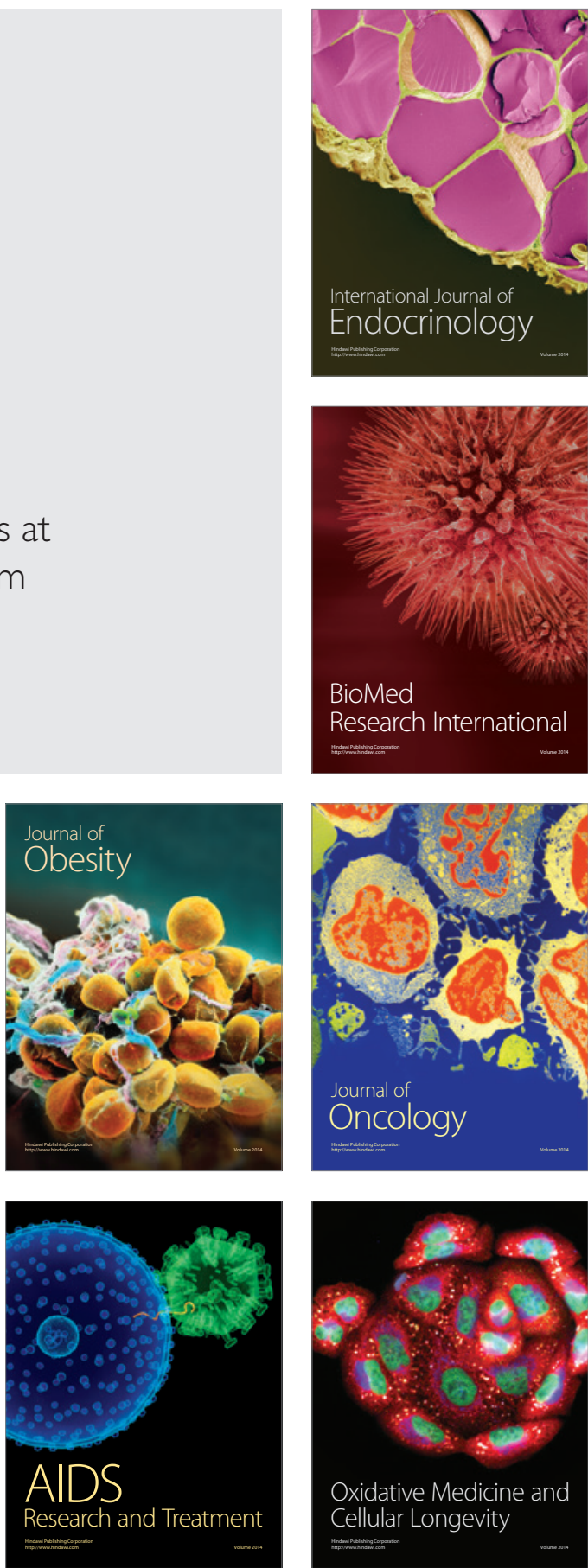